\title{
Las bacteriemias relacionadas con el catéter tunelizado de hemodiálisis y cuidados de enfermería
}

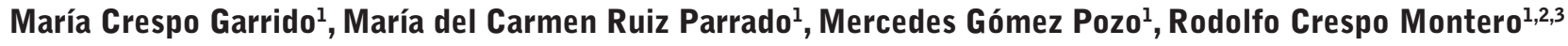 \\ ${ }^{1}$ Departamento de Enfermería. Facultad de Medicina y Enfermería. Universidad de Córdoba. Córdoba. España \\ ${ }^{2}$ Unidad de Gestión Clínica de Nefrología. Hospital Universitario Reina Sofía de Córdoba. Córdoba. España \\ ${ }^{3}$ Instituto Maimónides de Investigación Biomédica de Córdoba. Córdoba. España
}

\section{Resumen}

Introducción: En la actualidad, y debido a su avanzada edad y/o con comorbilidad diabética y cardiovascular asociada, un buen porcentaje de pacientes en hemodiálisis tienen que ser dializados mediante un catéter venoso central tunelizado.

Objetivo: Conocer la producción científica existente sobre la bacteriemia relacionada con el catéter venoso central tunelizado de hemodiálisis.

Material y Método: Se ha realizado una revisión sistemática mediante una búsqueda en las bases de datos SCOPUS, PubMed y ScieLo. Se incluyeron artículos escritos en inglés y español.

Resultados: Fueron revisados 28 artículos, entre los cuáles se incluían guías de práctica clínica, revisiones y estudios de investigación. Tras el análisis de los mismos, se evidenció que la bacteriemia relacionada con el catéter afecta a un número considerable de pacientes que portan un catéter venoso central tunelizado para hemodiálisis y se encontraron diferentes actitudes terapéuticas para abordarla. En cuanto a su prevención, se encontraron numerosas publicaciones sobre la efectividad de diferentes medidas farmacológicas, y aunque fueron menos las publicaciones encontradas acerca de la profilaxis no farmacológica, estas recalcaron la importancia del estricto cumplimiento de medidas de higiene y asepsia, como herramienta principal para prevenir su aparición y situaron a enfermería como elemento fundamental para cumplirlas.

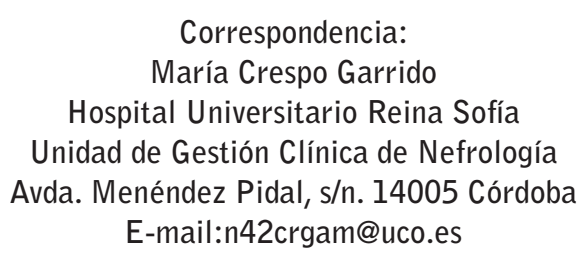

Conclusiones: Las medidas de higiene y asepsia constituyen la base de la prevención de la bacteriemia relacionada con el catéter, siendo la enfermería un factor clave para que estas se cumplan; y debido a que la mayor parte de la literatura se centra en las medidas farmacológicas, son necesarios más estudios que evidencien su importancia.

PALABRAS CLAVE: bacteriemia; catéter venoso central; hemodiálisis; prevención.

The bacteraemia to the tunnelled catheter of haemodialysis and nursing care

\section{Abstract}

Introduction: Currently, and due to advanced age and / or associated diabetic and cardiovascular comorbidity, a good percentage of haemodialysis patients must be dialysed through a tunnelled central venous catheter.

Objective: To know the existing scientific production on the bacteraemia related to the tunnelled central venous catheter of haemodialysis.

Material and Method: A systematic review has been carried out through a search of the SCOPUS, PubMed and ScieLo databases. Articles written in English and Spanish were included.

Results: 28 articles were reviewed, including clinical practice guidelines, reviews and research studies. After analysing them, it was evident that bacteraemia related to the catheter affects a considerable number of patients who have a tunnelled central venous catheter for haemodialysis and different therapeutic attitudes 
were found to address it. Regarding its prevention, numerous publications on the effectiveness of different pharmacological measures were found, and although there were fewer publications found about non-pharmacological prophylaxis, they emphasized the importance of strict compliance with hygiene and aseptic measures as the main tool to prevent its appearance and placing nursing as a fundamental element to consider.

Conclusions: Hygiene and aseptic measures are the basis for the prevention of catheter-related bacteraemia, being nursing a key factor in ensuring that these are complied with; and as much of the literature focuses on pharmacological measures, more studies are needed to demonstrate its importance.

KEYWORDS: bacteraemia; central venous catheter; Haemodialysis; prevention.

\section{Introducción}

La Enfermedad Renal Crónica afecta aproximadamente, a unos 4 millones de personas en nuestro país, de ahí su relevancia como problema de salud pública ${ }^{1,2}$. La incidencia de la Enfermedad Renal Crónica Avanzada (ERCA), que corresponde a los estadios 4 y 5 , está aumentando en países desarrollados entre el 5 y $10 \%$ anual ${ }^{3}$, debido al envejecimiento de la población y a la mayor prevalencia de Diabetes Mellitus (DM) tipo $\mathrm{II}^{4}$. En el estadio 5, estos pacientes van a necesitar un tratamiento renal sustitutivo (TRS): hemodiálisis (HD) diálisis peritoneal (DP) o trasplante renal (TR)2. Actualmente, se mantiene el aumento progresivo de DP como opción terapéutica inicial en el $16,9 \%$ de los casos, y del trasplante anticipado, que continua su tendencia de crecimiento, hasta suponer el $5 \%$ del total, pero sin duda, en la mayoría de pacientes que necesitan un TRS, la HD es la modalidad terapéutica más utilizada en nuestro país ${ }^{5}$, igual que ocurre en la mayoría de los países de nuestro entorno ${ }^{6}$.

Un elemento imprescindible para poder realizar una HD es el acceso vascular (AV), y aunque todas las evidencias científicas coinciden en que la fístula arteriovenosa autóloga ( $F A V a$ ) es la primera y mejor opción de $\mathrm{AV}$, existen circunstancias en las que no siempre es posible realizarla; teniendo que recurrir en estos casos a otro tipo de AV. EI AV de elección de segunda elección es la fístula arteriovenosa protésica $(F A V p)^{7-9}$. No obstante, y debido a que la gran mayoría de pacientes en HD son de avanzada edad y/o con comorbilidad diabética y cardiovascular asociada, lo que provoca que su lecho vascular no sea apto para una FAV, un buen porcentaje de ellos tienen que ser dializados mediante un catéter venoso central tunelizado (CVCT) ${ }^{10}$.

Estos catéteres, están pensados para tener una larga duración en el tiempo ${ }^{8}$. Sin embargo, su uso provoca con bastante frecuencia la aparición de complicaciones de origen mecánico o infeccioso. Entre las complicaciones mecánicas, se incluyen la trombosis intraluminal del catéter, pudiendo producir desde la oclusión del catéter hasta tromboembolismo pulmonar en casos más graves, y la estenosis venosa ${ }^{11}$.

En cuanto a las complicaciones infecciosas, se sitúan como las más frecuentes entre los pacientes con CVCT y pueden dividirse en locales o sistémicas ${ }^{12}$. Entre las locales, destacan la infección del orificio de salida y la infección del túnel o tunelitis ${ }^{13}$; y como complicaciones sistémicas se encuentra la Bacteriemia Relacionada con el Catéter (BRC), que es una de las principales causas de morbilidad, hospitalización y mortalidad entre los pacientes en $\mathrm{HD}^{14,15}$.

La BRC se define como un cuadro clínico de sepsis, sin otro foco aparente de infección, en el que se aísla el mismo microorganismo en hemocultivos simultáneos cuantitativos en una proporción superior o igual a 5:1 en las muestras extraídas a través del catéter respecto a las obtenidas por venopunción ${ }^{16}$. Además, la BRC puede derivar en complicaciones más graves como osteomielitis, endocarditis, tromboflebitis o espondilitis infecciosa, que pondrían poner la vida del paciente en grave peligro ${ }^{8}$. Como factores de riesgo para desarroIlar BRC se han señalado edad $>65$ años, DM y los niveles de albúmina $<3,5 \mathrm{mg} / \mathrm{dl}$; entre otros ${ }^{17}$.

Para el tratamiento de la BRC, existen diferentes medidas de abordaje, que van desde los antibióticos locales, hasta la retirada del catéter; centrándose las medidas de prevención en la utilización de medidas profilácticas farmacológicas ${ }^{14}$, y haciendo menor hincapié en los principios generales de la asepsia e higiene.

Sin embargo, no existe mucha bibliografía que documente el papel de los cuidados de enfermería en la prevención y/o incidencia de la BRC, motivo por el cual nos hemos planteado la presente revisión bibliográfica.

Por todo ello, nos planteamos como objetivo principal revisar la bibliografía existente sobre la bacteriemia relacionada con el CVCT para HD. 
Como objetivos específicos:

- Determinar las medidas de prevención de la BRC de HD.

- Analizar la influencia de los cuidados de enfermería en la prevención de la BRC de HD.

\section{Material y Método}

Se ha llevado a cabo una revisión bibliográfica sistemática. Para su elaboración se ha realizado una búsqueda en diferentes bases de datos, con la finalidad de recopilar la literatura científica más adecuada al tema abordado.

Las bases de datos consultadas han sido Pubmed, Scielo y Scopus, en el periodo de tiempo comprendido entre el 2 de marzo de 2017 y el 22 de mayo de 2017.

La estrategia de búsqueda consistió en introducir en cada base de datos las siguientes palabras clave: Bacteriemia ("Bacteremia"), Hemodiálisis ("Hemodialysis"), Catéter venoso central ("Central venous catheter"), Prevención ("Prevention"); y en las bases de datos con opción de tesauros fueron utilizados los siguientes: "Bacteremia", "Central venous catheters", "Renal dialysis", "Prevention and Control". Estos términos se asociaron entre sí mediante los operadores boleanos "and", "or" y "not", según conviniera, teniendo en cuenta la siguiente tabla.

\section{Los criterios de inclusión fueron:}

- Artículos originales completos y de revisión.

- Artículos que trataran la prevención de la BRCT, así como su influencia sobre esta.

- Artículos que analizaran la epidemiología de la BRCT en HD, así como la etiopatogénia.

- Artículos que compararan la eficacia de las distintas medidas terapéuticas ante BRC.

\section{Los criterios de exclusión fueron:}

- Artículos cuyo objeto de estudio fuesen pacientes pediátricos.

- Artículos que tratasen la bacteriemia en catéteres no tunelizados.

\section{Resultados}

\section{Selección de estudios preliminar}

Al introducir las combinaciones de palabras clave en cada base de datos se obtuvieron un total de 141 artículos, y mediante la lectura del título y resumen se descartaron 48 por cumplir algún criterio de exclusión, quedándonos con 93 artículos. Volviendo a revisar estos 93 más detalladamente, eliminamos los que aparecían en 20 más bases de datos, eligiendo Pubmed para descartar artículos que hubiésemos obtenido de aquí y, además, de más bases de datos, en total se eliminaron 25 artículos por duplicidad. Esto hacía que nos quedásemos con un total de 68 potencialmente adecuados para ser incluidos en nuestra revisión. Se volvió a revisar cada artículo, esta vez con más detenimiento, sobretodo en objetivos y resultados, y ya con más conocimiento sobre el tema abordado, haciendo una eliminación final de 41 artículos y seleccionando un total de 27 publicaciones para la

Para la selección de los artículos los criterios fueron que los distintos descriptores apareciesen en título, resumen o palabras clave, que hubiesen sido publicados desde 2007 hasta 2017. En las bases de datos que lo permitían, se limitaron los resultados introduciendo el filtro de texto completo, y en las que no, se descartaron aquellos artículos que no tuviesen texto completo mediante la revisión de los resultados. Se incluyeron artículos en inglés, portugués y castellano. elaboración de nuestra revisión, tal como se refleja en la Figura 1.

Además, se incluyó una Guía de Práctica Clínica sobre el AV de HD, que se encuentra "en prensa", pero que está disponible para todos los profesionales relacionados con los accesos vasculares para HD (8).

Los 28 artículos incluidos quedan reflejados en la Tabla 1, incluyendo: autor, año y país de la publicación, el tipo de estudio, así como la muestra estudiada en cada artículo. 


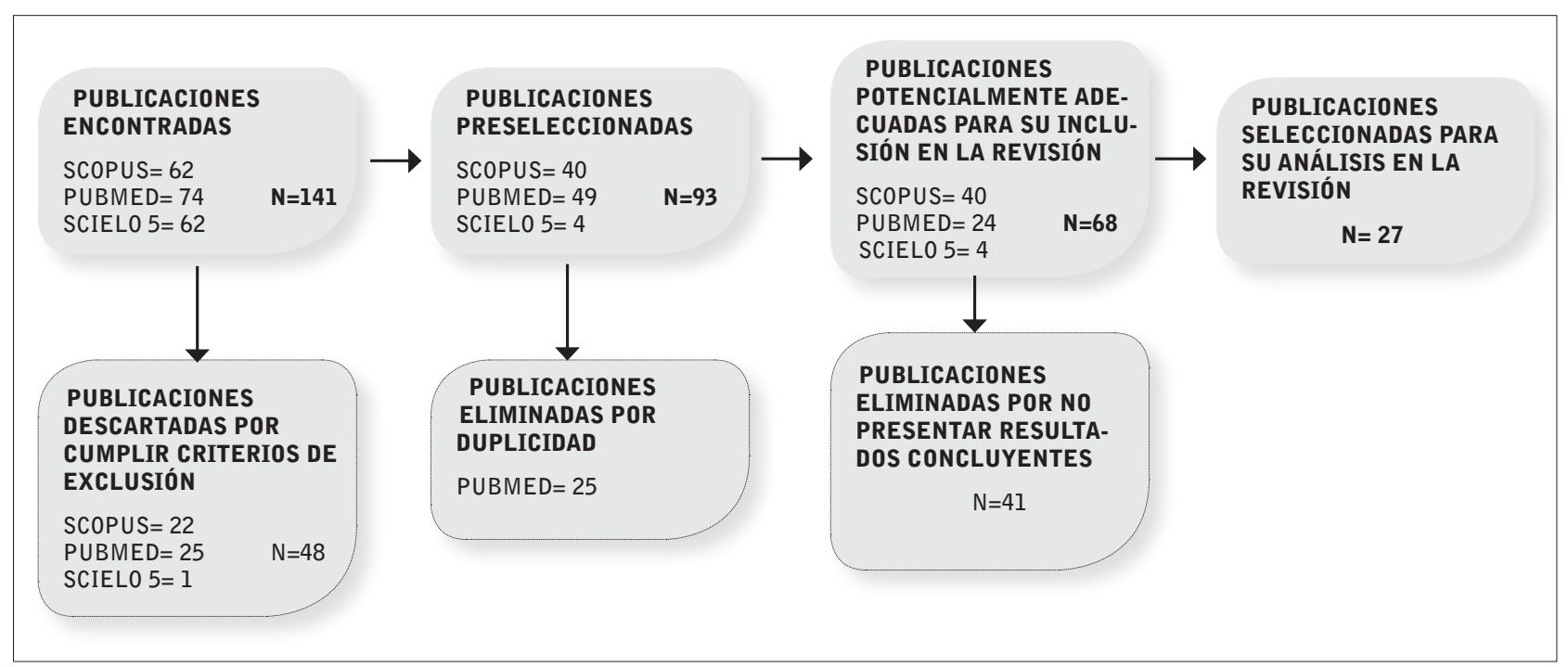

Figura 1. Diagrama de flujo.

Tabla 1. Artículos Revisados.

\begin{tabular}{|c|c|c|c|}
\hline AUTOR, AÑO, PAÍS & $\begin{array}{l}\text { TIPO DE } \\
\text { ESTUDIO }\end{array}$ & MUESTRA & CONCLUSIONES \\
\hline $\begin{array}{l}\text { Ramírez de Arellano M, Moreno T, Ibeas } \\
\text { J et al; 2016; España } \\
\text { JCR: Q4 } \\
\text { SJR: Q3 }\end{array}$ & $\begin{array}{c}\text { Guía de Práctica } \\
\text { Clínica }\end{array}$ & & $\begin{array}{l}\text { Recomendaciones sobre: } \\
\text { - Manipulación del CVCT. } \\
\text { - Seguimiento del CVCT. } \\
\text { - Complicaciones del CVCT. } \\
\text { - Infección relacionada con el CVCT. }\end{array}$ \\
\hline $\begin{array}{l}\text { McCann M, Moore ZEH; 2010; Irlan- } \\
\text { da }^{27} \\
\text { JCR: Q1 } \\
\text { SJR: Q1 }\end{array}$ & $\begin{array}{c}\text { Revisión } \\
\text { sistemática }\end{array}$ & $10^{*}$ & $\begin{array}{l}\text { La pomada de Mupirocina, la polisporina y la povidona io- } \\
\text { dada redujeron el riesgo de BRC, aunque no la mortalidad } \\
\text { relacionada con la infección. }\end{array}$ \\
\hline $\begin{array}{l}\text { Wang AY, Ivany JN, Perkovic V et al; } \\
\text { 2013; Australia }{ }^{28} \\
\text { JCR: Q1 } \\
\text { SJR: Q1 }\end{array}$ & $\begin{array}{c}\text { Revisión } \\
\text { sistemática y } \\
\text { meta-análisis }\end{array}$ & $28^{*}$ & $\begin{array}{l}\text { Las soluciones de citrato, soluciones antibióticas y el } \\
\text { activador tisular del plasminógeno reducen la incidencia } \\
\text { de BRC, pero ni la warfarina ni la heparina demostraron } \\
\text { efectos sobre la BRC. }\end{array}$ \\
\hline $\begin{array}{l}\text { Rabindranath KS, Bansal T, Adams J et } \\
\text { al; 2009; Reino Unido, EEUU29 } \\
\text { JCR: Q1 } \\
\text { SJR: Q1 }\end{array}$ & $\begin{array}{c}\text { Revisión } \\
\text { sistemática }\end{array}$ & $29 *$ & $\begin{array}{l}\text { La profilaxis con soluciones antibióticas intraluminales y } \\
\text { tópicas en el orificio de salida reduce la BRC, mientras que } \\
\text { los catéteres impregnados con antisépticos y la antibiote- } \\
\text { rapia sistémica perioperatoria no demostraron reducción } \\
\text { significativa. }\end{array}$ \\
\hline $\begin{array}{l}\text { Aslam S, Vaida F, Ritter M et al; 2014; } \\
\text { EEUU22 } \\
\text { JCR: Q1 } \\
\text { SJR: Q1 }\end{array}$ & $\begin{array}{c}\text { Revisión } \\
\text { sistemática y } \\
\text { meta-análisis }\end{array}$ & $28^{*}$ & $\begin{array}{l}\text { El intercambio del CVCT mediante guía o las soluciones } \\
\text { antibióticas de sellado tienen una significativa mayor } \\
\text { eficacia en el tratamiento de BRC en comparación a los } \\
\text { antibióticos sistémicos. }\end{array}$ \\
\hline $\begin{array}{l}\text { Zhao Y, Li Z, Zang L et al; 2014; China }{ }^{30} \\
\text { JCR: Q1 } \\
\text { SJR: Q1 }\end{array}$ & $\begin{array}{l}\text { Revisión } \\
\text { sistemática y } \\
\text { meta-análisis }\end{array}$ & $13^{*}$ & $\begin{array}{l}\text { Una solución de citrato sódico que contenga antibiótico es } \\
\text { más eficaz que el sellado de heparina en la prevención de } \\
\text { la BRC, mientras que el citrato por sí solo no muestra una } \\
\text { ventaja similar. }\end{array}$ \\
\hline
\end{tabular}




\begin{tabular}{|c|c|c|c|}
\hline AUTOR, AÑO, PAÍS & $\begin{array}{l}\text { TIPO DE } \\
\text { ESTUDIO }\end{array}$ & MUESTRA & CONCLUSIONES \\
\hline $\begin{array}{l}\text { Miler LM, Clark E, Dipchand C et al; } \\
\text { 2016; Canada }{ }^{21} \\
\text { JCR: Q1 } \\
\text { SJR: Q1 }\end{array}$ & $\begin{array}{c}\text { Revisión } \\
\text { bibliográfica }\end{array}$ & $\begin{array}{c}\text { No } \\
\text { especificada }\end{array}$ & $\begin{array}{l}\text { Staphylococcus aureus y coagulasa negativos causan entre } \\
\text { el } 40 \% \text { y } 80 \% \text { de BRC. } \\
\text { Endocarditis, osteomielitis, absceso epidural y artritis } \\
\text { séptica como complicaciones de la BRC. }\end{array}$ \\
\hline $\begin{array}{l}\text { Niyyar VD, Lock CE; 2013; Canada, } \\
\text { Estados Unidos }{ }^{31} \\
\text { JCR: Q1 } \\
\text { SJR: Q1 }\end{array}$ & $\begin{array}{l}\text { Revisión } \\
\text { bibliográfica }\end{array}$ & $\begin{array}{c}\text { No } \\
\text { especificada }\end{array}$ & $\begin{array}{l}\text { La gran variedad de soluciones de sellado, la diversidad } \\
\text { de pacientes y las limitaciones en el tamaño y diseño de } \\
\text { los estudios no permiten dar recomendaciones generales } \\
\text { respecto al uso del sellado del catéter de HD. }\end{array}$ \\
\hline $\begin{array}{l}\text { Aguinada A, del Pozo JL; } 2011 ; \\
\text { España }^{19} \\
\text { JCR: Q4 } \\
\text { SJR: Q3 }\end{array}$ & $\begin{array}{l}\text { Revisión } \\
\text { bibliográfica }\end{array}$ & $\begin{array}{c}\text { No } \\
\text { especificada }\end{array}$ & $\begin{array}{l}\text { Staphylococcus aureus y estafilococos coagulasa negati- } \\
\text { vos son los principales microorganismos implicados en la } \\
\text { infección del CVCT. } \\
\text { La estrategia preventiva fundamental para evitar la BRC } \\
\text { es la asepsia estricta. }\end{array}$ \\
\hline $\begin{array}{l}\text { Lok CE, Mokrzycki M H; } 2011 \text {; EE UU18 } \\
\text { JCR: Q1 } \\
\text { SJR: Q1 }\end{array}$ & $\begin{array}{l}\text { Revisión } \\
\text { bibliográfica }\end{array}$ & $\begin{array}{c}\text { No } \\
\text { especificada }\end{array}$ & $\begin{array}{l}\text { Las recientes disminuciones en la morbilidad infecciosa } \\
\text { relacionada con el catéter demuestran la eficacia de } \\
\text { las estrategias profilácticas y de manejo basadas en la } \\
\text { evidencia. }\end{array}$ \\
\hline $\begin{array}{l}\text { Boyce JM; 2012; EEUU32 } \\
\text { JCR: Q1 } \\
\text { SJR: Q1 }\end{array}$ & $\begin{array}{l}\text { Revisión } \\
\text { bibliográfica }\end{array}$ & $\begin{array}{c}\text { No } \\
\text { especificada }\end{array}$ & $\begin{array}{l}\text { Importancia de adoptar las intervenciones recomendadas } \\
\text { por los CDC para la prevención de BRC de diálisis. }\end{array}$ \\
\hline $\begin{array}{l}\text { Silva T, de Marchi D, Mendes M et al; } \\
\text { 2014; Brasil } \\
\text { JCR: Q3 } \\
\text { SJR: Q2 }\end{array}$ & $\begin{array}{l}\text { Revisión } \\
\text { bibliográfica }\end{array}$ & $\begin{array}{c}\text { No } \\
\text { especificada }\end{array}$ & $\begin{array}{l}\text { Los antibióticos tópicos y las soluciones de sellado del } \\
\text { catéter son los principales medios para prevenir la BRC, } \\
\text { aunque debe considerarse el riesgo de aparición de orga- } \\
\text { nismos resistentes a los antibióticos utilizados. }\end{array}$ \\
\hline $\begin{array}{l}\text { Crehuet Rodríguez I et al; 2013; } \\
\text { España }{ }^{33} \\
\text { JCR: - } \\
\text { SJR: Q4 }\end{array}$ & $\begin{array}{l}\text { Estudio } \\
\text { prospectivo y } \\
\text { comparativo }\end{array}$ & $49 * *$ & $\begin{array}{l}\text { El uso de bioconectores redujo casi un } 60 \% \text { la tasa de } \\
\text { BRC. Además, consiguieron eliminar el Staphylococcus } \\
\text { aureus como causante de BRC en la unidad. }\end{array}$ \\
\hline $\begin{array}{l}\text { Ashby DR, Power A, Singh S et al; 2009; } \\
\text { Reino Unido26 } \\
\text { JCR: Q1 } \\
\text { SJR: Q1 }\end{array}$ & $\begin{array}{l}\text { Estudio } \\
\text { prospectivo de } \\
\text { cohortes }\end{array}$ & $133 * *$ & $\begin{array}{l}\text { Ante una BRC, el tratamiento conservador del CVCT } \\
\text { mediante antibioterapia empírica sistémica tuvo éxito en } \\
\text { aproximadamente dos tercios de los casos. }\end{array}$ \\
\hline $\begin{array}{l}\text { Huddam B, Azak A, Koçak A et al; 2012; } \\
\text { Turquía }^{34} \\
\text { JCR: Q4 } \\
\text { SJR: Q3 }\end{array}$ & $\begin{array}{l}\text { Ensayo clínico } \\
\text { randomizado }\end{array}$ & $60 * *$ & $\begin{array}{l}\text { La profilaxis con antibióticos, previa a la inserción del } \\
\text { CVCT redujo significativamente la BRC y la pérdida del } \\
\text { catéter. }\end{array}$ \\
\hline $\begin{array}{l}\text { Maya ID, Carlton D, Estrada E et al; } \\
\text { 2007; Reino Unido, EEUU25 } \\
\text { JCR: Q1 } \\
\text { SJR: Q1 }\end{array}$ & $\begin{array}{l}\text { Estudio } \\
\text { observacional } \\
\text { prospectivo }\end{array}$ & $113 * *$ & $\begin{array}{l}\text { La terapia con sellado antibiótico de forma rutinaria no es } \\
\text { adecuada para pacientes con BRC causada por Staphylo- } \\
\text { coccus aureus. }\end{array}$ \\
\hline $\begin{array}{l}\text { Moore CL, Besarab A, Ajluni M et al; } \\
\text { 2014; EEUU35 } \\
\text { JCR: Q1 } \\
\text { SJR: Q1 }\end{array}$ & $\begin{array}{l}\text { Estudio } \\
\text { prospectivo } \\
\text { observacional de } \\
\text { cohortes }\end{array}$ & $555^{* *}$ & $\begin{array}{l}\text { El sellado profiláctico de gentamicina / citrato se asoció } \\
\text { con una reducción sustancial de la infección del torrente } \\
\text { sanguíneo relacionada con el CVCT. }\end{array}$ \\
\hline
\end{tabular}




\begin{tabular}{|c|c|c|c|}
\hline AUTOR, AÑO, PAÍS & $\begin{array}{l}\text { TIPO DE } \\
\text { ESTUDIO }\end{array}$ & MUESTRA & CONCLUSIONES \\
\hline $\begin{array}{l}\text { Moran J, Sun S, Khababa I et al; } 2011 ; \\
\text { EEUU36 } \\
\text { JCR: Q1 } \\
\text { SJR: Q1 }\end{array}$ & $\begin{array}{l}\text { Ensayo clínico } \\
\text { aleatorizado }\end{array}$ & $303 * *$ & $\begin{array}{l}\text { El sellado de rutina del CVCT con gentamicina } 320 \mu \mathrm{g} / \\
\mathrm{ml} \text { unida al citrato de sodio al } 4 \% \text { disminuye notablemente } \\
\text { la incidencia de BRC. }\end{array}$ \\
\hline $\begin{array}{l}\text { Oguzhan N, Pala C, Sipahioglu MH et } \\
\text { al; 2012; Turquía }{ }^{37} \\
\text { JCR: Q4 } \\
\text { SJR: Q3 }\end{array}$ & $\begin{array}{l}\text { Ensayo clínico } \\
\text { aleatorizado }\end{array}$ & $56 * *$ & $\begin{array}{l}\text { La solución de } \mathrm{NaCl} \text { al } 13 \% \text { y las soluciones de } \mathrm{NaCl} \text { más } \\
\text { hipertónicas revelaron potentes propiedades antimicrobia- } \\
\text { nas in vitro contra todos los microorganismos Gram-nega- } \\
\text { tivos aislados. }\end{array}$ \\
\hline $\begin{array}{l}\text { Power A, Duncan N, Singh SK et al; } \\
\text { 2009; Reino Unido } \\
\text { JCR: Q1 } \\
\text { SJR: Q1 }\end{array}$ & $\begin{array}{l}\text { Ensayo controlado } \\
\text { aleatorizado }\end{array}$ & $232 * *$ & $\begin{array}{l}\text { Debido a la falta de un efecto significativo en la infección } \\
\text { relacionada con el catéter y a la mayor tasa de efectos } \\
\text { adversos, el uso generalizado y a largo plazo del citrato al } \\
46,7 \% \text { no está justificado por este estudio. }\end{array}$ \\
\hline $\begin{array}{l}\text { Albalate M, Pérez García R, de Sequera } \\
\text { P et al; 2010; España }{ }^{39} \\
\text { JCR: Q4 } \\
\text { SJR: Q3 }\end{array}$ & $\begin{array}{l}\text { Estudio } \\
\text { observacional } \\
\text { retrospectivo }\end{array}$ & $32 * *$ & $\begin{array}{l}\text { El cumplimiento de medidas estrictas de asepsia durante } \\
\text { la manipulación del CVCT, es la principal herramienta } \\
\text { para evitar las BRC, sin que sean necesarias medidas } \\
\text { farmacológicas preventivas adicionales. }\end{array}$ \\
\hline $\begin{array}{l}\text { Kanaa M, Wright MJ, Bhandari S et al; } \\
\text { 2015; Reino Unido }{ }^{40} \\
\text { JCR: Q1 } \\
\text { SJR: Q1 }\end{array}$ & $\begin{array}{l}\text { Ensayo controlado } \\
\text { aleatorizado }\end{array}$ & $117^{* *}$ & $\begin{array}{l}\text { El citrato trisódico reduce significativamente la coloniza- } \\
\text { ción microbiana del CVCT en un } 87 \% \text { en comparación con } \\
\text { la heparina, pero la reducción de BRC no es significativa. }\end{array}$ \\
\hline $\begin{array}{l}\text { Moghaddas A, Abbasi MR, } \\
\text { Gharekhani A et al; 2015; Iran }{ }^{41} \\
\text { JCR: Q2 } \\
\text { SJR: Q1 }\end{array}$ & $\begin{array}{l}\text { Ensayo clínico } \\
\text { aleatorizado }\end{array}$ & 87 ** & $\begin{array}{l}\text { La solución de cotrimoxazol para el sellado del CVCT es } \\
\text { eficaz para reducir la incidencia de BRC y prolongar la } \\
\text { supervivencia del catéter en pacientes con HD. }\end{array}$ \\
\hline $\begin{array}{l}\text { Rosenblum A, Wang W, Ball LK et al; } \\
\text { 2014; EEUU42 } \\
\text { JCR: Q1 } \\
\text { SJR: Q1 }\end{array}$ & $\begin{array}{l}\text { Ensayo clínico } \\
\text { aleatorizado }\end{array}$ & $422 * *$ & $\begin{array}{l}\text { Con torundas de clorhexidina al } 2 \% \text { en solución alcohólica } \\
\text { ( } 70 \% \text { ) y compresas con } 70 \% \text { de alcohol para la limpieza } \\
\text { del orificio del catéter se redujeron las tasas de BRC. }\end{array}$ \\
\hline $\begin{array}{l}\text { Silva J, Costa T, Baptista A et al; 2008; } \\
\text { Portugal }{ }^{43} \\
\text { JCR: Q2 } \\
\text { SJR: Q2 }\end{array}$ & $\begin{array}{l}\text { Ensayo clínico } \\
\text { prospectivo, } \\
\text { aleatorizado }\end{array}$ & $116^{* *}$ & $\begin{array}{l}\text { La eficacia del sellado antibiótico es superior a la de los } \\
\text { antibióticos tópicos en la profilaxis de la BRC, y el uso de } \\
\text { ambos en el mismo CVCT no se asoció con efectos aditivos. }\end{array}$ \\
\hline $\begin{array}{l}\text { Silva TNV, Mendes ML, Abrao JMG et } \\
\text { al; 2012; Brasil }{ }^{44} \\
\text { JCR: Q3 } \\
\text { SJR: Q3 }\end{array}$ & $\begin{array}{l}\text { Estudio } \\
\text { observacional } \\
\text { prospectivo }\end{array}$ & $233^{* *}$ & $\begin{array}{l}\text { El sellado con cefazolina /gentamicina puede reducir la } \\
\text { tasa de BRC, sin asociación entre el sellado antibiótico y la } \\
\text { aparición de cepas resistentes. }\end{array}$ \\
\hline $\begin{array}{l}\text { Battistella M, Bhola C, Lock CE; } 2011 \text {; } \\
\text { Canada }^{23} \\
\text { JCR: Q1 } \\
\text { SJR: Q1 }\end{array}$ & $\begin{array}{l}\text { Estudio } \\
\text { observacional } \\
\text { prospectivo }\end{array}$ & $228^{* *}$ & $\begin{array}{l}\text { El uso a largo plazo de una solución poliantibiótica tópica } \\
\text { en el orificio de salida del } \mathrm{CVCT} \text {, junto con una cuidadosa } \\
\text { vigilancia, es una estrategia profiláctica eficaz contra la } \\
\text { BRC. }\end{array}$ \\
\hline $\begin{array}{l}\text { Abid S, De Silva S, Warwicker P et al; } \\
\text { 2008; Reino Unido }{ }^{24} \\
\text { JCR: Q3 } \\
\text { SJR: Q2 }\end{array}$ & $\begin{array}{l}\text { Estudio } \\
\text { observacional } \\
\text { retrospectivo }\end{array}$ & $358^{* *}$ & $\begin{array}{l}\text { La espondilitis infecciosa es una complicación poco } \\
\text { frecuente pero devastadora en pacientes con HD, con una } \\
\text { mortalidad del } 46 \% \text {. }\end{array}$ \\
\hline
\end{tabular}

Muestra: ${ }^{*}=$ pacientes; ${ }^{* *}=$ artículos. JCR: Journal Citation Report. SJR: SCimago Journal\& Country Rank. Q: Cuartil. BRC: Bacteriemia Relacionada con el Catéter. CVCT: Catéter Venoso Central Tunelizado. CDC: Centers for Disease Control and Prevention. HD: Hemodiálisis. 


\section{Descripción de los resultados}

En relación a la bibliografía descrita en la anterior tabla, se procede a clasificar los resultados en función de las siguientes variables.

\section{INCIDENCIA}

La BRC presenta una incidencia muy variable; y sobre todo cambiante a lo largo de los años. Las series publicadas hace unos años (Lok et al y Aguinada $A$ et al, 2011) reflejaban una incidencia que oscilaba entre 0.6 y 6.5 episodios por 1000 días de utilización de CVCT ${ }^{18,19}$.

Sin embargo, actualizaciones más recientes publicaban un rango de 2,5 a 5 episodios por 1000 días de utilización de catéter, lo cual se corresponde con una incidencia de 0,9 a 2 episodios de BRC por catéter y año ${ }^{8}$.

\section{ETIOPATOGENIA}

La base etiológica de la BRC se debe a un proceso gradual que comienza con la adherencia de microorganismos y colonización de la superficie interna del catéter, hasta la formación de un biofilm o película bacteriana madura, que consiste en una comunidad de microorganismos autosostenible alojada en la superficie interna del catéter y protegida por una matriz de exopolisacáridos secretada por los propios microorganismos. Este biofilm maduro va a desarrollar una alta resistencia a los antibióticos sistémicos, dificultando la erradicación de la BRC ${ }^{18,20}$.

La mayoría de la literatura revisada, coincide en que los organismos Gram-positivos son los responsables de la mayoría de las BRC en HD ${ }^{8,18,19,21-23}$. Dentro de estos, el Staphylococcus aureus se sitúa como el coco positivo que causa más BRC, seguido muy de cerca por el Staphylococcus coagulasa-negativa. Estos 2 gérmenes, son los responsables de entre el $40 \%$ y $80 \%$ de la BRC totales. El Staphylococcus aureus resistente a la meticilina (MRSA) también aparece con frecuencia $^{8,18,19,21,22}$.

Además de tener una alta incidencia, la BRC producida por Staphylococcus aureus está asociada en un 30$50 \%$ a mayor número de complicaciones metastásicas graves y mortalidad $8,18,19,21$, y un riesgo cuatro veces mayor de bacteriemia recidivante en comparación con otros microorganismos ${ }^{18}$.
También se han descrito BRC causadas por Staphylococcus epidermis, Streptococcus spp., Enterococcus spp. y Corynebacterium spp, y bacilos Gram-negativos como Pseudomonas/Stenotrophemonas, aunque con menos frecuencia que los organismos primeramente descritos 8,18,19,22. No obstante, en los últimos años se está viendo aumentada la BRC por gramnegativos, pudiendo llegar a representar en algunos centros hasta el $30-40 \% 8$.

\section{FACTORES DE RIESGO}

Los principales factores de riesgo asociados a la BRC de HD se han señalado, la higiene inadecuada del paciente, BRC previas, hospitalizaciones previas recientes, pautas de diálisis inadecuadas, hipoalbuminemia, DM, estatus inmunológico comprometido, hipertensión arterial (HTA) y arteroesclerosis ${ }^{18}$.

\section{COMPLICACIONES DERIVADAS DE LA BRC}

Las complicaciones derivadas de la BRC pueden ser graves, y más concretamente las complicaciones infecciosas metastásicas, que aparecen con una frecuencia de entre el $5 \%$ al $34 \%$, y que en algunos casos pueden llegar a ocasionar la muerte ${ }^{8,18,21}$.

Se ha señalado a la endocarditis, como la complicación más frecuente derivada de la BRC. Como la complicación más frecuente en segundo lugar se ha encontrado la osteomielitis vertebral o espondilitis infecciosa ${ }^{8,18,21}$. Respecto a esta complicación, el estudio de Abid et al, reveló una incidencia de 1 episodio de espondilitis por cada 119 pacientes con CVCT y año, mostrando además las consecuencias tan devastadoras que supuso en los pacientes que la desarrollaron, dando lugar a una mortalidad del $46 \%{ }^{24}$.

Además, se describen como complicaciones frecuentes, aunque menos que las dos anteriores, el shock séptico, la embolia séptica en encéfalo y pulmón principalmen$\mathrm{te}^{8,18}$, y la artritis séptica ${ }^{18,21}$. Entre las complicaciones descritas como muy poco comunes se cita el absceso epidural espinal18,21.

La mortalidad derivada de la BRC, por tanto, se produce como complicación de alguno de los cuadros clínicos mencionados anteriormente y presenta una incidencia alta, con rangos que oscilan desde el $5 \%$ al $34 \%$ de los $\operatorname{casos}^{8,18,21}$. 


\section{DIAGNÓSTICO DE LA BRC}

La sospecha y el diagnóstico de la BRC se ha basado en la presencia de manifestaciones clínicas, locales y/o sistémicas de infección. Por eso, la aparición de signos generales de infección como fiebre, escalofríos e inestabilidad hemodinámica, y otros más específicos, como presencia de exudado e inflamación local en el orificio de salida cutáneo del CVCT, pone de manifiesto la posible $B R C^{8,19,21}$.

Sin embargo, los signos clínicos aislados se consideran insuficientes, por lo que para confirmar el diagnóstico de BRC, es necesario realizar hemocultivos de sangre periférica y del catéter y que en ambos aparezca aislado el mismo microorganismo en una proporción superior o igual a 5:1 en las muestras extraídas a través del catéter respecto a las obtenidas por venopunción ${ }^{8,19}$.

\section{ABORDAJE TERAPEÚTICO}

Para abordar la BRC se han identificado diferentes estrategias de tratamiento, que dependen de la presentación clínica de la BRC, del microorganismo aislado, y las opciones alternativas de acceso vascular del paciente ${ }^{8,21}$, y se enumeran a continuación:

\section{Retirada del CVCT}

Las guías y evidencias describen como criterios para retirar el CVCT la presencia de alguna de estas circunstancias ${ }^{8,19}$ :

- Infección local complicada (tunelitis asociada a fiebre o exudado purulento en el punto de inserción).

- Persistencia de fiebre o bacteriemia 48-72 horas después de haber iniciado antibiótico adecuado a la sensibilidad de los microorganismos.

- Evidencia de infección metastásica.

- Aislamiento de microorganismos muy virulentos: Staphylococcus aureus, Pseudomonas spp, Candida spp o microorganismos multirresistentes.

\section{Tratamiento conservador del CVCT}

El tratamiento conservador mediante sellado antibiótico del CVCT asociado a antibioterapia sistémica es utilizado con frecuencia y numerosas publicaciones le han otorgado un porcentaje de éxito alto. Además, to- das coinciden en que el sellado antibiótico junto con antibioterapia sistémica presenta mayores índices de curación que los antibióticos sistémicos solos ${ }^{8,19,22,25}$. Sin embargo, los mismos autores ${ }^{22,26}$, que encuentran altas tasas de éxito con este tratamiento, señalaban como limitaciones que el sellado antibiótico junto con la antibioterapia sistémica habían fracasado en la mayoría de BRC producidas por Staphylococcus aureus, originándose numerosas recidivas de la infección.

De acuerdo a este hallazgo en concreto, aparecen estudios como el de Maya et al $^{25}$, en el que utilizaron el sellado antibiótico unido a la antibioterapia sistémica para tratar BRC causada por Staphylococcus aureus, y concluye desaconsejando el tratamiento antibiótico conservador por no ser efectivo ante este microorganismo, refutando así las recomendaciones dadas por las guías ${ }^{8}$.

\section{Recambio del CVCT a través de una guía}

Una alternativa aceptable al tratamiento conservador con antibióticos en BRC no complicadas es el recambio del CVCT mediante una guía, ya que con esta opción terapéutica se elimina definitivamente la biocapa del interior del catéter causante de la infección. Además, permite conservar el lugar de implantación del catéter, algo especialmente útil en pacientes con el lecho vascular deteriorado, ${ }^{8,22}$.

\section{PREVENCION Y CUIDADOS DE ENFERMERIA}

Se han encontrado numerosas publicaciones referentes a la prevención de la BRC en $H^{8,18,20,23,27-44}$.

Existen estudios sobre medidas preventivas anteriores a la inserción del CVCT: el ensayo clínico de Huddam et $\mathrm{a}^{34}$ documentó que la profilaxis con antibióticos, previa a la inserción del CVCT redujo significativamente la BRC y la pérdida del catéter. Por otro lado, una revisión sistemática ${ }^{29}$ concluyó que los catéteres impregnados con antisépticos y la antibioterapia sistémica durante la implantación del catéter no mostraron reducción significativa. Hay otros autores que directamente han abordado la prevención de la BRC revisando los factores que disminuyen la utilización del CVCT, encontrando que la designación de al menos una enfermera especialista en el AV en las unidades de HD, redujo la utilización de CVCT y por tanto la infección relacionada con el mismo ${ }^{18}$. 
Para los pacientes que ya portan un CVCT se ha descrito un amplio rango de diferentes medidas profilácticas: soluciones antimicrobianas tópicas 8,20,23,27,29,43; sellado del catéter con antibióticos $28,41,44$ sustancias anticoagulantes ${ }^{28,30,38,40}$ con la combinación de ambas $^{30,31,35,36}$ o con otras sustancias ${ }^{37}$; uso de dispositivos como los bioconectores ${ }^{33}$ y medidas universales de asepsia y uso de protocolos $8,19,32,39$.

El uso de soluciones antimicrobianas tópicas ha sido calificado por diversos autores como una de las mejores medidas profilácticas para la $B R C^{8,20,23,27,29}$. Sin embargo, Silva et al documentaron que la eficacia del sellado antibiótico es superior a la de los antibióticos tópicos como medida profiláctica de la BRC, además añadió que el uso de ambos en el mismo CVCT no se asoció con efectos aditivos ${ }^{43}$.

En relación al sellado profiláctico del CVCT, la evidencia demuestra que en general, la combinación de citrato sódico y antibióticos es la más eficaz para prevenir la $B R C^{8,28,30,35,36}$, aunque otras publicaciones también refieren buenos resultados para el sellado solo con antibióticos $29,41,44$. Por su parte, el citrato solo u otros anticoagulantes como la heparina no refieren efectos significativos en la prevención de $B \mathrm{RC}^{28,38,40}$.

El sellado con otras sustancias como la solución de $\mathrm{NaCl}$ hipertónico reveló importantes propiedades antimicrobianas ${ }^{37}$.

Sin embargo, la revisión de Niyyar et al ${ }^{31}$ de 2013 concluye con que debido a la gran variedad de soluciones de sellado, la diversidad de pacientes y las limitaciones en el tamaño y diseño de los estudios no permiten dar recomendaciones generales respecto al uso del sellado del catéter de HD.

Sobre el uso de bioconectores solamente encontramos un estudio ${ }^{33}$ y reporta que su uso originó una reducción del casi $60 \%$ de la BRC en CVCT.

Con respecto a la asepsia para prevenir la BRC, un estudio mostró tasas muy bajas de BRC mediante el cumplimiento de medidas estrictas de asepsia durante la manipulación del CVCT, sin que fuesen necesarias medidas farmacológicas preventivas adicionales. Para ello, se realizó una formación continuada del personal de enfermería ${ }^{39}$.

En esta misma línea, otras revisiones, recalcan la importancia de adoptar las intervenciones recomendadas por los Centers for Disease Control and Preven- tion (CDC) para la prevención de BRC de diálisis y la importancia de la asepsia estricta, como estrategia preventiva fundamental para evitar la BRC ${ }^{19,32}$.

Por último, el documento más actualizado, La Guía Española del Acceso Vascular, realiza una serie de Recomendaciones enfocadas a preservar la asepsia, entre las que se incluyen la correcta higiene de manos, y que las conexiones y desconexiones sean realizadas únicamente por enfermería especializada en unidades de diálisis, siendo necesarias dos personas, una de ellas enfermera ${ }^{8}$.

\section{Discusión}

El objetivo de este estudio fue conocer la bibliografía existente sobre la BRC en HD, pero sobre todo, su relación con los cuidados y las medidas de prevención desde el punto de vista de enfermería.

$\mathrm{Su}$ incidencia es muy variable, aunque se evidencia una disminución en los últimos estudios revisados, coincidente con la disminución del uso de catéteres para HD en los últimos años, y sobre todo a las recomendaciones de las guías ${ }^{8,45}$, pues como se ha publicado por algunos autores en series anteriores al periodo de revisión, existía mayor incidencia, hospitalizaciones y mortalidad en los pacientes con CVCT en HD, relacionadas con la $B \mathrm{RC}^{47}$.

Respecto a los gérmenes más frecuentes causantes de la BRC, se ha señalado al Staphylococcus aureus y al Staphylococcus coagulasa-negativa, responsables de entre el $40-80 \%$ del total del BRC en el paciente en $H D^{8,18,19,21,22,23 ;}$; aunque no son los únicos. Algunos autores han descrito episodios polimicrobianos, 0 incluso episodios de BRC causados por micobacterias u hongos ${ }^{46}$.

Como principales factores de riesgo asociados a la $B R C$ de HD se han descrito higiene inadecuada, BRC previas, hospitalizaciones previas, hipoalbuminemia, DM, estatus inmunológico comprometido, hipertensión arterial (HTA) y arteroesclerosis18, aunque algunos autores solo han encontrado relación con las hospitalizaciones anteriores ${ }^{48}$.

En cuanto a las complicaciones de la BRC, cabe destacar sobre todo, las complicaciones. infecciosas metastásicas, siendo la endocarditis, y osteomielitis vertebral o espondilitis infecciosa las más frecuen- 
tes derivada de la $B R C^{8,18,21}$. También se han descrito como complicaciones frecuentes, aunque menos que las anteriores, el shock séptico, la embolia séptica en encéfalo y pulmón principalmente ${ }^{8,18}$, y la artritis séptica ${ }^{18,21}$, siendo estas entidades infecciosas las responsables de la mortalidad derivada de la BRC, que puede oscilar entre el $5 \%$ al $34 \%$ de los $\operatorname{casos}^{8,18,21}$.

Por otro lado, hay que destacar que aunque el diagnóstico de la BRC no era un objetivo principal de nuestro estudio, se ha encontrado abundante bibliografía al respecto $0^{8,19,21}$, y aunque existe un consenso sobre los signos clínicos del mismo, algunos autores destacan que en ocasiones no está claro el diagnóstico ${ }^{49,50}$. Por ello, las últimas guías aconsejan la confirmación mediante hemocultivos de sangre periférica y del catéter y que en ambos aparezca aislado el mismo microorganismo ${ }^{8}$.

Un apartado importante revisado, ha sido sin duda, el abordaje terapéutico, proponiéndose distintas estrategias, según la presentación clínica de la BRC, el microorganismo aislado, y las opciones alternativas de acceso vascular del paciente ${ }^{8,21}$. La retirada del catéter, el tratamiento conservador del mismo o el recambio del CVCT a través de una guía ${ }^{8,19}$, son las medidas aconsejadas. De todos ellos, y teniendo en cuenta sus indicaciones, el más controvertido es el tratamiento conservador del catéter, puesto que se han empleado diferentes procedimientos y pautas de tratamiento, siendo el sellado antibiótico del CVCT asociado a antibioterapia sistémica el más utilizado8,19,22,25; aunque presenta limitaciones en la mayoría de BRC producidas por Staphylococcus aureus 22,26 .

La última variable ha sido la más determinante para nuestra revisión. Se ha observado múltiples estrategias profilácticas para la BRC, aunque sin embargo, la mayor parte de la literatura se ha centrado en comprobar la efectividad de los recursos farmacológicos. No obstante, se han revisado diferentes publicaciones que defienden las medidas asépticas y de higiene como la base fundamental para evitar la BRC, y la mayoría de ellas explicitan la necesidad de cuidados de enfermería que mantengan la asepsia estricta durante el manejo del CVCT, para lo que se resalta como instrumento principal la formación específica de los enfermeros que vayan a estar en contacto con CVCT.

Es por ello, por lo que pensamos que es imprescindible la implicación de enfermería en la aplicación de las recomendaciones basadas en la evidencia que recogen algunas guías, como garantía de cuidados de calidad, y que aseguren la seguridad del paciente.

\section{Limitaciones del estudio}

Como principales limitaciones del estudio, señalar que la mayoría de artículos y revisiones encontradas, se centran en investigaciones médicas, derivadas de la epidemiología, etiología, diagnóstico, complicaciones y tratamiento farmacológico de la BRC.

Sin embargo, existe muy poca bibliografía que documente el papel de los cuidados de enfermería en la prevención de la BRC. Si existen, muchas recomendaciones sobre la necesidad de cuidar los aspectos preventivos desde el punto de vista de la higiene y la asepsia, pero no se encuentran originales de investigación de enfermería que aborden este objetivo de estudio.

\section{Consideraciones prácticas}

A pesar de todas las medidas terapéuticas recomendadas, y de las diferentes estrategias desarrolladas para evitar la BRC, como el sellado preventivo, etc; pensamos que lo más importante es seguir las recomendaciones de las Guías elaboradas recientemente, porque son las que recogen la última evidencia, y porque en concreto en la española, se le dedica un apartado muy especial, a los cuidados de enfermería y como se debe manejar el CVCT para HD.

\section{Conclusiones}

La BRC es un cuadro clínico que aparece con una frecuencia relativamente elevada en pacientes portadores de CVCT. Sin embargo, su incidencia ha sido cambiante, disminuyendo durante los últimos años. Como los microorganismos más frecuentes se situan el Staphylococcus aureus y coagulasa-negativa, responsables de producir la mayoría de los casos de BRC.

Su aparición está asociada a numerosos factores de riesgo, entre ellos patologías crónicas como DM e HTA, y situaciones como BRC previas u hospitalizaciones recientes del paciente. Además, puede derivar en complicaciones metastásicas graves que, en algunos casos, llegan a poner en riesgo la vida del paciente.

Para confirmar su diagnóstico, ante la presencia de signos y síntomas de infección, son necesarios hemocultivos, y para su abordaje existen diversas medidas terapéuticas. 
En cuanto a la prevención de la BRC existe una gran variedad de medidas farmacológicas, pero las medidas de higiene y asepsia constituyen una herramienta imprescindible para disminuirla. Debido a que la mayor parte de la literatura se centra en la profilaxis farmacológica, se hace evidente la necesidad de otorgarle a estas medidas no farmacológicas la importancia que merecen.

Además la enfermería desarrolla un papel fundamental en la prevención mediante medidas de higiene y asepsia, ya que es la que está en constante contacto con el CVCT y es la que puede asegurar que estas se cumplen de manera estricta.

\section{El autor declara que no hay conflicto de interés.}

Recibido: 12 septiembre 2017

Revisado: 20 septiembre 2017

Modificado: 28 septiembre 2017

Aceptado: 2 octubre 2017

\section{Bibliografía}

1. De Francisco AL, Sanjuán F, Foraster A, et al. Estudio epidemiológico de pacientes ancianos con insuficiencia renal crónica en hemodiálisis. Nefrología 2008;28:48-53.

2. Informe de Diálisis y Trasplante. Registro Español de Enfermos Renales. 2015. Sociedad Española de Nefrología. [Consultado 15 mayo 2017]. Disponible en: http://www.senefro.org/contents/webstructure/ reer0viedo2016.pdf.

3. EDTA-ERA Registry 2014. European Renal Association - European Dialysis and Transplant Association. [Consultado 20 mayo 2017]. Disponible en: https://www.era-edta-reg.org/files/ annualreports/pdf/AnnRep2014.pdf.
4. Aitken E, Geddes C, Thomson P, Kasthuri R, Chandramohan $\mathrm{M}$, Berry $\mathrm{C}$ et al. Immediate access arteriovenous grafts versus tunnelled central venous catheters: study protocol for a randomised controlled trial. BioMed Central 2015; 16:42.

5. Accesovascularparahemodiálisis.SociedadEspañola de Nefrología. 2016. [Consultado 19 mayo 2017]. Disponible en: http://www.senefro.org/modules. php? name $=$ webstructure \&idwebstructure $=36$.

6. Niyyar VD, Chan MR. Interventional Nephrology: Catheter Dysfunction-Prevention and Troubleshooting. Clin J Am Soc Nephrol 2013; 8: 1234-1243.

7. Saxena AK, Panhotra BR. Prevention of catheterrelated bloodstream infections: An appraisal of developments in designing an infection-resistant 'dream dialysis-catheter'. NEPHROLOGY 2005; 10: 240-248.

8. Liangos 0 , Gul A, Madias NE, Jaber BL. Long-Term Management of the Tunneled Venous Catheter. Seminars in Dialysis 2006; 19:158-164.

9. Allon M, Daugirdas J, Depner TA, Greene T, Ornt $D$, Schwab SJ. Effect of change in vascular access on patient mortality in hemodialysis patients. Am J Kidney Dis. 2006;47(3):469.

10. García P, Payá E, Olivares R, Cotera A, Rodríguez $J$, Sanz M. Diagnóstico de las infecciones asociadas a catéteres vasculares centrales. Rev Chil Infect 2003; 20 (1): 41-50.

11. Akoh JA. Vascular Access Infections: Epidemiology, Diagnosis, and Management. Curr Infect Dis Rep 2011 ; 13:324-332.

12. Carrasco Salinas $C$, Ruiz García M, Gómez López $V E$, Méndez Ordóñez R, López Andreu M, Crespo Montero R. Análisis de las hospitalizaciones por bacteriemia relacionada con el catéter de hemodiálisis. Enferm Nefrol 2013; 16 (2): 8892.

13. Palomar Martínez $M$, Álvarez Lerma F, Riera Badía MA, León Gil C, López Pueyo MJ, Díaz Tobajas $C$ et al. Prevención de la bacteriemia relacionada con catéteres en UCI mediante una intervención multifactorial. Informe del estudio piloto. Med Intensiva 2010; 34(9):581-589. 
14. Powe NR, Jaar B, Furth SL, Hermann J, Briggs W. Septicemia in dialysis patients: incidence, risk factors, and prognosis. Kidney Int 1999; 55: 10811090.

15. Lok CE, Mokrzycki MH. Prevention and management of catheter-related infection in hemodialysis patients. Kidney Int $2011 ; 79,587-$ 598.

16. Aguinada $A$, del Pozo JL. Infección asociada a catéter en hemodiálisis: diagnóstico, tratamiento y prevención. NefroPlus 2011; 4(2):1-10.

17. Silva T, de Marchi D, Mendes ML, Barretti P, Ponce D. Approach to prophylactic measures for central venous catheter-related infections in hemodialysis: A critical review. Hemodialysis International 2014; 18:15-23.

18. Miler LM, Clark E, Dipchand C, Hiremath S, Kappel $J$, Kiaii M et al. Hemodialysis Tunneled CatheterRelated Infections. Canadian Journal of Kidney Health and Disease 2016; 3: $1-11$.

19. Aslam S, Vaida F, Ritter M, Metha RL. Systematic Review and Meta-Analysis on Management of Hemodialysis Catheter-Related Bacteremia. J Am Soc Nephrol 2014; 25:1-15.

20. Battistella M, Bhola C, Lock CE. Long-term Follow-up of the Hemodialysis Infection Prevention With Polysporin Ointment (HIPPO) Study: A Quality Improvement Report. Am J Kidney Dis. 2011; 57(3):432-441 doi:10.1053/j. ajkd.2010.10.009.

21. Abid S, De Silva S, Warwicker P, Farrington K. Infective spondylodiscitis in patients on highflux hemodialysis and on-line hemodiafiltration. Hemodial Int 2008 0ct; 12(4):463-470.

22. Maya ID, Carlton D, Estrada E, Allon M. Treatment of Dialysis Catheter-Related Staphylococcus aureus Bacteremia With an Antibiotic Lock: A Quality Improvement Report. American Journal of Kidney Diseases 2007; 50(2):289-295.

23. Ashby DR, Power $A$, Singh $S$, Choi $P$, Taube $D H$, Duncan ND et al. Bacteremia Associated with Tunneled Hemodialysis Catheters: Outcome after Attempted Salvage. Clin J Am Soc Nephrol 2009; 4:1601-1605.
24. McCann M, Moore ZEH. Interventions for preventing infectious complications in haemodialysis patients with central venous catheters. Cochrane Database of Systematic Reviews 2010, Issue 1. Art. No.: CD006894.

25. Wang AY, Ivany JN, Perkovic V, Gallagher MP, Jardine MJ. Anticoagulant therapies for the prevention of intravascular catheters malfunction in patients undergoing haemodialysis: systematic review and meta-analysis of randomized, controlled trials. Nephrol Dial Transplant (2013) 28: 28752888.

26. Rabindranath KS, Bansal T, Adams J, Das R, Shail R, MacLeod AM et al. Systematic review of antimicrobials for the prevention of haemodialysis catheter-related infections. Nephrol Dial Transplant (2009) 24: 3763-3774.

27. Zhao Y, Li Z, Zhang L, Yang J, Yang Y, Tang Y et al. Citrate Versus Heparin Lock for Hemodialysis Catheters: A Systematic Review and Meta-analysis of Randomized Controlled Trials. Am J Kidney Dis 2014; 63(3):479-490.

28. Niyyar VD, Lock CE. Pros and cons of catheter lock solutions. Curr Opin Nephro Hypertens 2013; 22:669-674.

29. Boyce JM. Prevention of Central Line-Associated Bloodstream Infections in Hemodialysis Patients. Infect Control Hosp Epidemiol 2012;33(9):936944. doi: 10.1086/667369.

30. Crehuet Rodríguez I, Bernárdez Lemus MA, Ramírez Crehuet $\mathrm{M}$ et al. Bioconectores: ¿Son realmente eficaces en la reducción de las bacteriemias relacionadas con el catéter permanente para hemodiálisis?. Enferm Nefrol 2013; 16 (4): 235-240.

31. Huddam B, Azak A, Koçak A, Ortabozkoyun L, Duranay M.The Efficacy of Prophylactic Antibiotics Administration prior to Insertion of Tunneled Catheter in Hemodialysis Patients. Renal Failure 2012; 34(8): 998-1001.

32. Moore CL, Besarab A, Ajluni M et al. Comparative Effectiveness of Two Catheter Locking Solutions to Reduce Catheter-Related Bloodstream Infection in Hemodialysis Patients. Clin J Am SocNephrol2014; 9:1232-1239. 
33. Moran J, Sun S, Khababa I, Pedan A, Doss S, Schiller B. A Randomized Trial Comparing Gentamicin/ Citrate and Heparin Locks for Central Venous Catheters in Maintenance Hemodialysis Patients. Am J Kidney Dis. 59(1):102-107.

34. Oguzhan. Locking Tunneled Hemodialysis Catheters with Hypertonic Saline $(26 \% \mathrm{NaCl})$ and Heparin to Prevent Catheter-Related Bloodstream Infections and Thrombosis: A Randomized, Prospective Trial. Renal Failure 2012, 34(2): 181-188.

35. Power A, Duncan N, Singh SK et al. Sodium Citrate Versus Heparin Catheter Locks for Cuffed Central Venous Catheters: A Single-Center Randomized Controlled Trial. Am J Kidney Dis 2009; 53:1034104.

36. Albalate M, Pérez García $R$, De Sequera P, Alcazar $\mathrm{R}$, Puerta M, Ortega $M$ et al. ¿Hemos olvidado lo más importante para prevenir las bacteriemias en pacientes portadores de catéteres para hemodiálisis?.Nefrologia2010;30(5):573-7.

37. Kanaa M, Wright MJ, Akbani $H$, Laboi P, Bhandari S, Sandoe JA. Cathasept Line Lock and Microbial Colonization of Tunneled Hemodialysis Catheters: A Multicenter Randomized Controlled Trial. Am J Kidney Dis 2015.

38. Moghaddas A, Abbasi MR, Gharekhani A, DasthiKhavidaki S, Razeghi E, Jafari $A$ et al. Prevention of hemodialysis catheter related bloodstream infections using a cotrimoxazole-lock technique. Future Microbiol 2015; 10(2):169-178.

39. Rosenblum A, Wang W, Ball LK, Latham C, Maddux FW, Lacson EJr. Hemodialysis Catheter Care Strategies: A Cluster Randomized Quality Improvement Initiative. Am J Kidney Dis 2014; 63(2):259-267.

40. Silva J, Costa T, Baptista A, Ramos A, Ponce P. Catheter-Related Bacteremia in Hemodialysis:
Which Preventive Measures to Take?. Nephron Clin Pract 2008;110:c251-c257.

41. Silva TN, Mendes ML, Abrão JM, Caramori JT, Ponce D. Successful prevention of tunneled central catheter infection by antibiotic lock therapy using cefazolin and gentamicin. Int Urol Nephrol 2012.

42. NKF KDOKI Guidelines. Clinical practice guidelines for vascular access for Vascular Access; 2006. [Consultado 06 junio 2017]. Disponible en: http:// www2.kidney.org/professionals/KD0QI/guideline upHD_PD_VA/va_rec7.htm.

43. Mokrzycki MH, Zhang M, Cohen H, Golestaneh $L$, Laut JM, Rosenberg S0. Tunneled haemodialysis catheter bacteraemia: risk factors for bacteraemia recurrence, infectious complications and mortality. Nephrol Dial Transplant 2006;21(4):1024-31.

44. Epidemiology, Surveillance, and Prevention of Bloodstream Infections in Hemodialysis Patients. American Journal of Kidney Diseases 2010; 56(3):566-577.

45. Fram D, Okuno Pinto MF. Risk factors for bloodstream infection in patients at a Brazilian hemodialysis center: a case-control study. BMC Infectious Diseases 2015; 15:158.

46. Gauna TT, Oshiro E, Luzio $Y C$, Paniago AMM, Pontes ERJC, Chang MR. Bloodstream infection in patients with end-stage renal disease in a teaching hospital in central-western Brazil. Ver. Soc. Bras. Med. Trop. 2013; 46(4):426-432.

47. Vanholder R, Canaud $B$, Fluck $R$, Jadoul $M$, Labriola $L$, Marti-Monros A, et al. Diagnosis, prevention and treatment of haemodialysis catheter related bloodstream infections. (CRBSI): apposition Statement of European Renal Best Practice (ERBP). Nephr Dial Trans Plus 2010; 3:234-246.

Este artículo se distribuye bajo una Licencia Creative Commons Atribución-NoComercial 4.0 Internacional. https://creativecommons.org/licenses/by-nc/4.0/ 